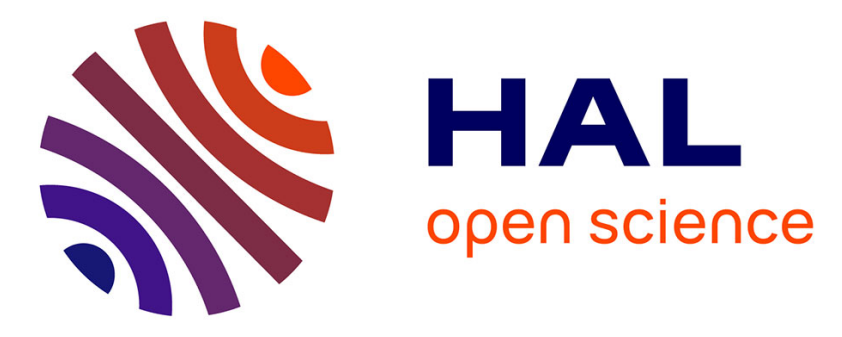

\title{
Linear Theory of Shells Taking into Account Surface Stresses
}

Holm Altenbach, Victor A. Eremeyev, Nikita F. Morozov

\section{To cite this version:}

Holm Altenbach, Victor A. Eremeyev, Nikita F. Morozov. Linear Theory of Shells Taking into Account Surface Stresses. Doklady Physics, 2009, 54 (12), pp.531-535. hal-00824864

\section{HAL Id: hal-00824864 \\ https://hal.science/hal-00824864}

Submitted on 22 May 2013

HAL is a multi-disciplinary open access archive for the deposit and dissemination of scientific research documents, whether they are published or not. The documents may come from teaching and research institutions in France or abroad, or from public or private research centers.
L'archive ouverte pluridisciplinaire HAL, est destinée au dépôt et à la diffusion de documents scientifiques de niveau recherche, publiés ou non, émanant des établissements d'enseignement et de recherche français ou étrangers, des laboratoires publics ou privés. 


\title{
Linear Theory of Shells Taking into Account Surface Stresses
}

\author{
H. Altenbach ${ }^{a}$, V. A. Eremeyev ${ }^{b}$, and Academician N. F. Morozov
}

Recently, the theory of elasticity with surface stresses [1] was developed for nanomechanical problems. Within this theory, the surface stresses $\tau$ act on the body boundary or its portion alongside with the stress tensor $\sigma$ defined in its volume $V$ and on its surface $\Omega$. The tensor $\tau$ generalizes the scalar surface tension known in hydromechanics in the case of solids. The introduction of surface stresses makes it possible to describe, in particular, the size effect characteristic for nanomaterials.

Mathematical investigations of surface stresses in solids and liquids go back to the works of Laplace, Young, Gibbs, etc. (see, for example, review [2]). The foundations of mechanics of solids with consideration of the surface stresses were developed in [3-5], etc. From the mechanical viewpoint, the model of surface stresses proposed in [3] is equivalent to that for a deformable body with an elastic membrane glued on its surface, and the tensor $\tau$ can be considered as the stress resultants tensor operating in this membrane. Model [3] is generalized in [5] to the case of taking into account the bending stiffness of the body surface and actually reduced to the presence of a shell on the body surface described by the equations of a theory of the Kirchhoff-Love type.

As applied to mechanical problems of objects such as nanodimensional beams, plates, and shells, the continuum approaches based on the Kirchhoff-Love, Reissner-Mindlin, etc., models of plates and shells are widely used in the literature. In this case, a substantial element of the theory is the modification of constitutive equations with taking into account the behavior features of nanomaterials. In particular, the problems of mechanics of nanodimensional plates

\footnotetext{
${ }^{a}$ Martin Luther University, Halle-Wittenberg, D-06099 Germany

${ }^{b}$ Southern Scientific Center, Russian Academy of Sciences, Rostov-on-Don, 344090 Russia

e-mail:eremeyev.victor@gmail.com

${ }^{c}$ St. Petersburg State University, St. Petersburg, 198904 Russia
}

with taking into account the surface stresses were discussed in [6-12].

In this work, generalizing the results [12] to the case of the linear theory of elastic shells taking into account the transverse shear, we obtained the equations of equilibrium and constitutive equations for the stress resultants and couples tensors while taking into account the surface stresses acting on the shell surfaces. The effective shell stiffness, in particular, the bending stiffness $D_{\text {eff }}$, depends here also on the surface elastic moduli, which is substantial for nanodimensional thicknesses.

1. For the formulation of boundary-value problems of the theory of elasticity with surface stresses, we use the variational method. Let the elastic body occupy the region $V \in \mathbb{R}^{3}$ bounded by a smooth surface $\Omega$. Without restriction of generality, we assume that the displacement-vector field $\mathbf{u}$ is zero at the boundary portion $\Omega_{1} \subset \Omega$ :

$$
\left.\mathbf{u}\right|_{\Omega_{1}}=\mathbf{0},
$$

and, at the remaining boundary portion $\Omega_{2}=\Omega \backslash \Omega_{1}$, the external loads $\varphi$ are set, and the surface stresses $\tau$ act.

Further, for simplicity, we restrict ourselves to the consideration of an isotropic body. The strain energy density of an elastic body is given by the formula

$$
\begin{gathered}
W=W(\varepsilon) \equiv \frac{1}{2} \lambda \operatorname{tr}^{2} \varepsilon+\mu \varepsilon \cdot \varepsilon, \\
\varepsilon=\varepsilon(\mathbf{u}) \equiv \frac{1}{2}\left(\nabla \mathbf{u}+\nabla \mathbf{u}^{T}\right),
\end{gathered}
$$

where $\varepsilon$ is the strain tensor, $\lambda$ and $\mu$ are the Lamé constants, $\nabla$ is the spatial gradient operator, the dot designates the scalar product, $\varepsilon \cdot \varepsilon=\operatorname{tr}(\varepsilon \cdot \varepsilon)$, tr is the tracecalculation operator.

For an isotropic body, the surface energy $U$ set on $\Omega_{2}$ can be written as [3]

$$
\begin{gathered}
U=U(\boldsymbol{\epsilon}) \equiv \tau_{0} \operatorname{tr} \epsilon+\frac{1}{2} \lambda_{S} \operatorname{tr}^{2} \epsilon+\mu_{S} \epsilon \cdot \boldsymbol{\epsilon}, \\
\boldsymbol{\epsilon}=\boldsymbol{\epsilon}(\mathbf{u}) \equiv \frac{1}{2}\left(\left(\nabla_{S} \mathbf{u}_{S}\right) \cdot \mathbf{A}+\mathbf{A} \cdot\left(\nabla_{S} \mathbf{u}_{S}\right)^{T}\right) .
\end{gathered}
$$




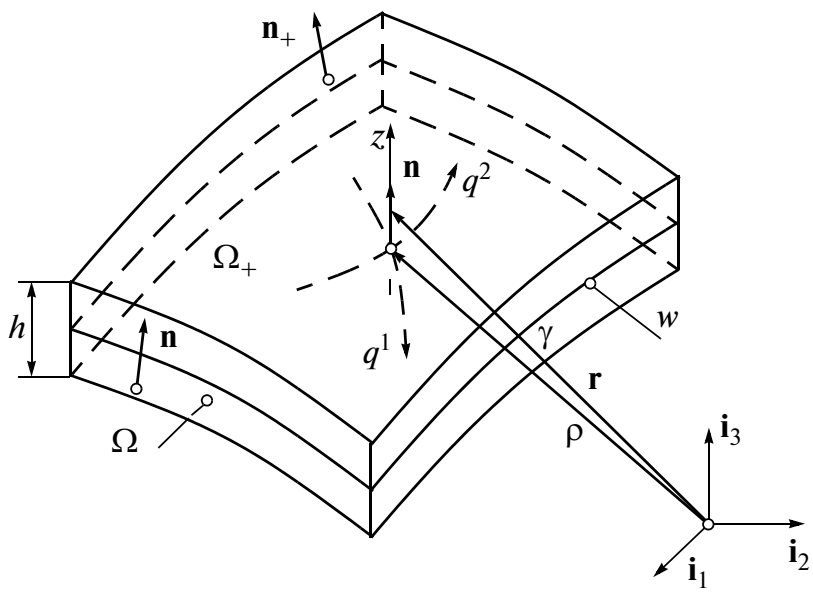

Fig. 1. Shell-like body.

Here, $\epsilon$ is the surface strain tensor, $\mathbf{u}_{S}=\left.\mathbf{u}\right|_{\Omega_{2}}$ is the displacement vector on $\Omega_{2}, \nabla_{S}$ is the surface gradient operator [13], $\mathbf{A}=\mathbf{I}-\mathbf{n} \otimes \mathbf{n}, \mathbf{I}$ is the three-dimensional unit tensor, $\mathbf{n}$ is the external-normal vector to $\Omega_{2}, \lambda_{S}$, and $\mu_{S}$ are the surface elastic moduli (the analogues of the Lamé surface constants), and $\tau_{0}$ is the residual surface stress. The function $U$ in Eq. (2) coincides with the strain energy of a membrane-shell, and $\epsilon$ is equal to the linear stretch tensor [14].

The total-energy functional for a body with the surface stresses has the form

$$
\begin{gathered}
J(\mathbf{u})=E(\mathbf{u})-A(\mathbf{u}), \\
E(\mathbf{u})=\int_{V} W(\varepsilon) d V+\int_{\Omega_{2}} U(\boldsymbol{\epsilon}) d \Omega, \\
A(\mathbf{u})=\int_{V} \mathbf{f} \cdot \mathbf{u} d V+\int_{\Omega_{2}} \varphi \cdot \mathbf{u} d \Omega .
\end{gathered}
$$

Here, $\mathbf{f}$ is the vector of external volume forces.

The stationarity condition $\delta J(\mathbf{u})=0, \forall \delta \mathbf{u}:\left.\delta \mathbf{u}\right|_{\Omega_{1}}=$ $\mathbf{0}$, leads to the equilibrium equations and the static boundary conditions:

$$
\nabla \cdot \sigma+\mathbf{f}=\mathbf{0},\left.\quad\left(\mathbf{n} \cdot \sigma-\nabla_{S} \cdot \tau\right)\right|_{\Omega_{2}}=\varphi .
$$

The stress and surface-stress tensors in Eq. (3) are given by formulas

$$
\begin{gathered}
\sigma=\frac{\partial W}{\partial \boldsymbol{\varepsilon}}=\lambda \mathbf{I t r} \boldsymbol{\varepsilon}+2 \mu \boldsymbol{\varepsilon}, \\
\tau=\frac{\partial U}{\partial \boldsymbol{\epsilon}}=\tau_{0} \mathbf{A}+\lambda_{S} \mathbf{A} \operatorname{tr} \boldsymbol{\epsilon}+2 \mu_{S} \boldsymbol{\epsilon} .
\end{gathered}
$$

It is also possible to show that an arbitrary solution of boundary-value problem (1) and (3) is the stationary point of the functional $J(\mathbf{u})$ on the kinematically admissible displacement fields $\mathbf{u}$.
Further, we require fulfillment of the conditions of positive definiteness of the functions $W(\varepsilon)$ and $U(\epsilon)$ :

$$
W(\varepsilon)>0 \quad \forall \boldsymbol{\varepsilon} \neq \mathbf{0}, \quad U(\boldsymbol{\epsilon})>0 \quad \forall \boldsymbol{\epsilon} \neq \mathbf{0} .
$$

The fulfillment of inequalities (4) results in the following restrictions on the elastic moduli:

$$
\mu>0, \quad 3 \lambda+2 \mu>0, \quad \mu_{S}>0, \quad \mu_{S}+\lambda_{S}>0 .
$$

It is necessary to note that assumptions (4) should be fulfilled independently from each other. If, in particular, we assume that $U<0$ for certain deformations, or inequalities $(5)_{3,4}$ are violated, it is possible to show that $J(\mathbf{u})$ proves to be unbounded from below.

2. For the transition to the shell-theory equations, we consider a three-dimensional body, one of the characteristic sizes of which is much less than others - the so-called shell-like body (Fig. 1). The shelllike body volume $V$ is bounded by two faces $\Omega_{ \pm}$and the lateral surface $\Omega_{v}$. We introduce also a middle (base) surface $\omega$, which is equidistant from $\Omega_{ \pm}$. The lateral surface $\Omega_{v}$ represents the ruled surface formed by the motion of the normal $\mathbf{n}$ to $\omega$ along its contour $\gamma \equiv \partial \omega$. It is convenient to present the radius vector $\mathbf{r}$ of the shell-body points as [13, 14]

$$
\mathbf{r}=\rho\left(q^{1}, q^{2}\right)+z \mathbf{n},
$$

where $\rho$ is the radius vector of points of the base surface $\omega, \mathbf{n}$ is the vector of the normal to $\omega, z$ is the coordinate counted from the normal to $\omega, z \in[-h / 2, h / 2], h$ is the shell thickness, and $q^{1}$ and $q^{2}$ are the Gaussian coordinates at $\omega$. The radius vectors of $\Omega_{ \pm}$are $\mathbf{r}_{ \pm}=\rho \pm \mathbf{n} h / 2$, respectively.

We display certain auxiliary formulas related to the description of tensor fields near the surface $\omega[13,14]$. The basic and dual bases and the surface nabla operator at $\omega$ are given by the formulas

$$
\begin{array}{cc}
\rho_{\alpha}=\frac{\partial \rho}{\partial q^{\alpha}}, & \rho_{\alpha} \cdot \rho^{\beta}=\delta_{\alpha}^{\beta}, \quad \alpha, \beta=1,2, \\
\nabla_{S}=\rho^{\alpha} \frac{\partial}{\partial q^{\alpha}},
\end{array}
$$

where $\delta_{\alpha}^{\beta}$ is the Kronecker delta. We use the quantities $q^{1}, q^{2}$, and $z$ as the curvilinear coordinates in the vicinity of $\omega$. Then the following formulas hold:

$$
\begin{gathered}
\mathbf{r}_{\alpha}=\frac{\partial \mathbf{r}}{\partial q^{\alpha}}=\rho_{\alpha}+z \frac{\partial \mathbf{n}}{\partial q^{\alpha}}=(\mathbf{A}-z \mathbf{B}) \cdot \boldsymbol{\rho}_{\alpha}, \\
\mathbf{r}_{3}=\mathbf{r}^{3}=\mathbf{n}, \\
\mathbf{r}^{\alpha}=(\mathbf{A}-z \mathbf{B})^{-1} \cdot \rho^{\alpha}, \quad \mathbf{r}_{\alpha} \cdot \mathbf{r}^{\beta}=\delta_{\alpha}^{\beta}, \\
\mathbf{B}=-\nabla_{S} \mathbf{n}, \\
\nabla=\mathbf{r}^{\alpha} \frac{\partial}{\partial q^{\alpha}}+\mathbf{n} \frac{\partial}{\partial z}=(\mathbf{A}-z \mathbf{B})^{-1} \cdot \nabla_{S}+\mathbf{n} \frac{\partial}{\partial z} \\
\nabla_{S}^{ \pm}=\left(\mathbf{A}+\frac{h}{2} \mathbf{B}\right)^{-1} \cdot \rho^{\alpha} \frac{\partial}{\partial q^{\alpha}}=\left(\mathbf{A}+\frac{h}{2} \mathbf{B}\right)^{-1} \cdot \nabla_{S},
\end{gathered}
$$


$(\mathbf{A}-z \mathbf{B})^{-1} \cdot(\mathbf{A}-z \mathbf{B})=(\mathbf{A}-z \mathbf{B}) \cdot(\mathbf{A}-z \mathbf{B})^{-1}=\mathbf{A}$, where $\mathbf{B}$ is the curvature tensor of the surface $\omega$.

We assume that the following surface stresses act on $\Omega_{2}=\Omega_{+} \cup \Omega_{-}$:

$$
\left.\left(\mathbf{n}_{ \pm} \cdot \sigma+\nabla_{S}^{ \pm} \cdot \tau_{S}^{ \pm}\right)\right|_{\Omega_{ \pm}}=\varphi_{ \pm} .
$$

In Eq. (6), $\mathbf{n}_{ \pm}$are the vectors of the normal to $\Omega_{ \pm}$(see Fig. 1), $\tau_{S}^{ \pm}$and $\varphi_{ \pm}$are the surface stresses and surface loads at $\Omega_{ \pm}$, respectively,

$$
\begin{aligned}
\tau_{ \pm} & =\tau_{0}^{ \pm} \mathbf{A}+\lambda_{S}^{ \pm} \mathbf{A} \operatorname{tr} \epsilon_{ \pm}+2 \mu_{S}^{ \pm} \epsilon_{ \pm}, \\
2 \epsilon_{ \pm} & =\left(\nabla_{S}^{ \pm} \mathbf{u}_{S}^{ \pm}\right) \cdot \mathbf{A}+\mathbf{A} \cdot\left(\nabla_{S}^{ \pm} \mathbf{u}_{S}^{ \pm}\right)^{T} .
\end{aligned}
$$

Here, $\mu_{S}^{ \pm}$and $\lambda_{S}^{ \pm}$are the surface elastic moduli, and $\tau_{0}^{ \pm}$are the residual stresses at $\Omega_{ \pm}$. We note that the surface gradient operators $\nabla_{S}^{ \pm}$at $\Omega_{ \pm}$, differ from each other, in general.

For passing to the two-dimensional equations of shell theory, we use a procedure known in shell theory - the integration of equilibrium equations (3) over thickness with taking into account boundary conditions (6) used also in [12]. Integrating Eqs. (3) over $z$ with taking into account Eq. (6), we obtain

$$
\nabla_{S} \cdot \mathbf{T}+G_{+} \nabla_{S}^{+} \cdot \tau_{+}+G_{-} \nabla_{S}^{-} \cdot \tau_{-}+\mathbf{q}=\mathbf{0},
$$

where $\mathbf{T}=\left\langle(\mathbf{A}-z \mathbf{B})^{-1} \cdot \sigma\right\rangle$ is the stress resultants tensor, $\mathbf{q}=G_{+} \varphi_{+}-G_{-} \varphi_{-}+\langle\mathbf{f}\rangle$ is the surface density of the external forces acting to the shell,

$$
\begin{gathered}
\langle(\ldots)\rangle=\int_{-h / 2}^{h / 2}(\ldots) G d z, \quad G=G(z) \equiv \operatorname{det}(\mathbf{A}-z \mathbf{B}), \\
G_{ \pm}=G\left( \pm \frac{h}{2}\right) .
\end{gathered}
$$

Taking the vector product of equilibrium equation (3) and $z \mathbf{n}$ at the left and integrating over the thickness, we obtain the second equilibrium equation

$$
\begin{gathered}
\nabla_{S} \cdot \mathbf{M}+\mathbf{T}_{\times}+\mathbf{m}+\frac{h}{2} G_{+} \mathbf{n} \times \nabla_{S}^{+} \cdot \tau_{+} \\
-\frac{h}{2} G_{-} \mathbf{n} \times \nabla_{S}^{-} \cdot \tau_{-}=\mathbf{0},
\end{gathered}
$$

The tensor $\mathbf{M}=-\left\langle(\mathbf{A}-z \mathbf{B})^{-1} \cdot z \boldsymbol{\sigma} \times \mathbf{n}\right\rangle$ is the stress couples tensor, while

$$
\mathbf{m}=\frac{h}{2} G_{+} \mathbf{n} \times \varphi_{+}+\frac{h}{2} G_{-} \mathbf{n} \times \varphi_{-}+\langle z \mathbf{n} \times \mathbf{f}\rangle
$$

is the surface moment distributed over $\omega$, the subscript $\times$ designates the vector invariant of the secondorder tensor, in particular, the vector invariant is calculated for the dyad formed by the vectors $\mathbf{a}$ and $\mathbf{b}$ from the formula $(\mathbf{a} \otimes \mathbf{b})_{\times}=\mathbf{a} \times \mathbf{b}$.

The presence of terms related to the fact that $\tau_{ \pm}$distinguishes equilibrium Eqs. (7) and (8) from the equilibrium equations of the linear theory of shells. Trans- forming them while taking into account the assumption that $\mathrm{h}\|\mathbf{B}\| \ll 1$, we obtain

$$
\nabla_{S} \cdot \mathbf{T}^{*}+\mathbf{q}=\mathbf{0}, \quad \nabla_{S} \cdot \mathbf{M}^{*}+\mathbf{T}_{\times}^{*}+\mathbf{m}=\mathbf{0},
$$

where the effective stress resultants and couples tensors $\mathbf{T}^{*}$ and $\mathbf{M}^{*}$ are introduced

$$
\begin{gathered}
\mathbf{T}^{*}=\mathbf{T}+\mathbf{T}_{S}, \quad \mathbf{M}^{*}=\mathbf{M}+\mathbf{M}_{S}, \\
\mathbf{T}_{S}=\tau_{+}+\tau_{-}, \quad \mathbf{M}_{S}=-\frac{h}{2}\left(\tau_{+}-\tau_{-}\right) \times \mathbf{n} .
\end{gathered}
$$

For description the shell deformations, we assume the displacement-field approximation linear in thickness used in the theory of plates and shells with taking into account the transverse shear (see, for example, [15]):

$$
\begin{gathered}
\mathbf{u}\left(q^{1}, q^{2}, z\right)=\mathbf{w}\left(q^{1}, q^{2}\right)-z \vartheta\left(q^{1}, q^{2}\right), \\
\mathbf{n} \cdot \vartheta=0 .
\end{gathered}
$$

Here it is assumed that the rotation vector $\vartheta$ is kinematically independent of the displacement vector of the shell midsurface w. Equation (11) leads to the formulas

$$
\mathbf{u}_{S}^{ \pm}=\mathbf{w}+\frac{h}{2} \vartheta, \quad \epsilon_{ \pm}=\epsilon+\frac{h}{2} \kappa,
$$

where

$$
\begin{aligned}
& \boldsymbol{\epsilon}=\frac{1}{2}\left(\nabla_{S} \mathbf{w} \cdot \mathbf{A}+\mathbf{A} \cdot\left(\nabla_{S} \mathbf{w}\right)^{T}\right), \\
& \kappa=\frac{1}{2}\left(\nabla_{S} \vartheta \cdot \mathbf{A}+\mathbf{A} \cdot\left(\nabla_{S} \vartheta\right)^{T}\right)
\end{aligned}
$$

are the two-dimensional tensors of extension-shear and bending-torsion deformations. Using Eq. (12), we obtain for the surface stresses $\tau_{ \pm}$the expressions

$$
\tau_{ \pm}=\tau_{0}^{ \pm} \mathbf{A}+\lambda_{ \pm}^{S} \mathbf{A} \operatorname{tr} \epsilon+2 \mu_{ \pm}^{S} \epsilon+\frac{h}{2}\left(\lambda_{ \pm}^{S} \mathbf{A} \operatorname{tr} \kappa+2 \mu_{ \pm}^{S} \kappa\right) .
$$

In the case of the shell with identical surface properties, i.e., when $\tau_{0}^{+}=\tau_{0}^{-}=\tau_{0}, \mu_{+}^{S}=\mu_{-}^{S}=\mu_{S}, \lambda_{+}^{S}=\lambda_{-}^{S}=\lambda_{S}$, we obtain the stress resultants and couples tensors generated by the surface-stress action:

$$
\begin{gathered}
\mathbf{T}_{S}=2 \tau_{0} \mathbf{A}+C_{1}^{S} \epsilon+C_{2}^{S} \mathbf{A} \operatorname{tr} \boldsymbol{\epsilon}, \\
\mathbf{M}_{S}=-\left[D_{1}^{S} \kappa+D_{2}^{S} \mathbf{A t r} \kappa\right] \times \mathbf{n}, \\
C_{1}^{S}=4 \mu^{S}, \quad C_{2}^{S}=2 \lambda^{S}, \quad D_{1}^{S}=h^{2} \mu^{2}, \\
D_{2}^{S}=\frac{h^{2} \lambda^{S}}{2} .
\end{gathered}
$$

With taking into account Eqs. (10), it follows from Eq. (13) that the surface stresses render no effect on the transverse shear forces because $\mathbf{T}_{S} \cdot \mathbf{n}=\mathbf{0}$ and have little or no effect on the stiffness and the transverse shear of the shell. From Eq. (13), it can be seen also that the residual surface stresses $\tau_{0}$ do not affect the shell stiffness, although, naturally they affect its stress state. 


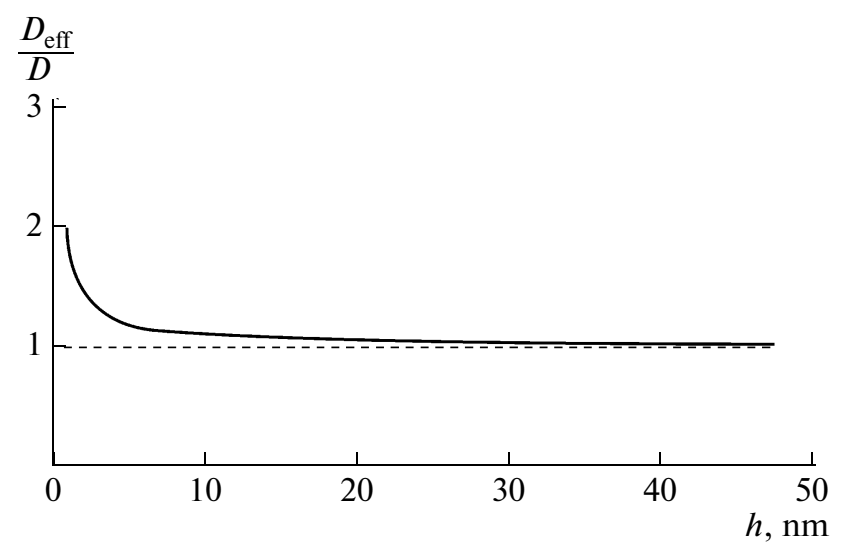

Fig. 2. Dependence of bending stiffness on thickness.

For $\mathbf{T}$ and $\mathbf{M}$, we accept the constitutive equations in one of the simplest forms given, for example, in [15]

$$
\begin{gathered}
\mathbf{T} \cdot \mathbf{A}-\frac{1}{2}(\mathbf{M} \cdot \mathbf{B}) \mathbf{A} \times \mathbf{n}=\frac{\partial W_{S}}{\partial \epsilon}, \\
\mathbf{T} \cdot \mathbf{n}=\frac{\partial W_{S}}{\partial \gamma}, \quad \mathbf{M}=\frac{\partial W_{S}}{\partial \boldsymbol{\kappa}}, \\
2 W_{S}=\epsilon \cdot \mathbf{C} \cdot \boldsymbol{\epsilon}+\boldsymbol{\kappa} \cdot \mathbf{D} \cdot \boldsymbol{\kappa}+\Gamma \boldsymbol{\gamma} \cdot \boldsymbol{\gamma} .
\end{gathered}
$$

Here, $W_{S}$ is the surface strain energy density, $\mathbf{C}$ and $\mathbf{D}$ are the fourth-order tensors determining the tangential and bending stiffness of the shell, $\gamma$ is the transverse-shear vector: $\gamma=\nabla_{S}(\mathbf{w} \cdot \mathbf{n})-\vartheta$, while $\Gamma$ is the transverse-shear stiffness. For an isotropic shell [15]

$$
\begin{aligned}
& \mathbf{C}=C_{11} \mathbf{a}_{1} \mathbf{a}_{1}+C_{22}\left(\mathbf{a}_{2} \mathbf{a}_{2}+\mathbf{a}_{4} \mathbf{a}_{4}\right), \\
& \mathbf{D}=D_{22}\left(\mathbf{a}_{2} \mathbf{a}_{2}+\mathbf{a}_{4} \mathbf{a}_{4}\right)+C_{33} \mathbf{a}_{3} \mathbf{a}_{3},
\end{aligned}
$$

where

$$
\begin{gathered}
\mathbf{a}_{1}=\mathbf{A} \equiv \mathbf{e}_{1} \otimes \mathbf{e}_{1}+\mathbf{e}_{2} \otimes \mathbf{e}_{2}, \\
\mathbf{a}_{2}=\mathbf{e}_{1} \otimes \mathbf{e}_{1}-\mathbf{e}_{2} \otimes \mathbf{e}_{2}, \\
\mathbf{a}_{3}=-\mathbf{A} \times \mathbf{n}=\mathbf{e}_{1} \otimes \mathbf{e}_{2}-\mathbf{e}_{2} \otimes \mathbf{e}_{1}, \\
\mathbf{a}_{4}=\mathbf{e}_{1} \otimes \mathbf{e}_{2}+\mathbf{e}_{2} \otimes \mathbf{e}_{1},
\end{gathered}
$$

$\mathbf{e}_{1}$ and $\mathbf{e}_{2}$ are the unit vectors lying in the plane tangent to $\omega\left(\mathbf{e}_{1} \cdot \mathbf{e}_{2}=\mathbf{e}_{1} \cdot \mathbf{n}=\mathbf{e}_{2} \cdot \mathbf{n}=0\right)$. The components $C_{11}$, $C_{22}, D_{22}, D_{33}$, and $\Gamma$ are given by the formulas [15]

$$
\begin{gathered}
C_{11}=\frac{E h}{2(1-v)}, \quad C_{22}=\frac{E h}{2(1+v)}, \\
D_{22}=\frac{E h^{3}}{24(1+v)}, \quad D_{33}=\frac{E h^{3}}{24(1-v)}, \quad \Gamma=k \mu h, \\
E=2 \mu(1+v), \quad v=\frac{\lambda}{2(\lambda+\mu)}, \\
C \equiv C_{11}+C_{22}=\frac{E h}{1-v^{2}},
\end{gathered}
$$

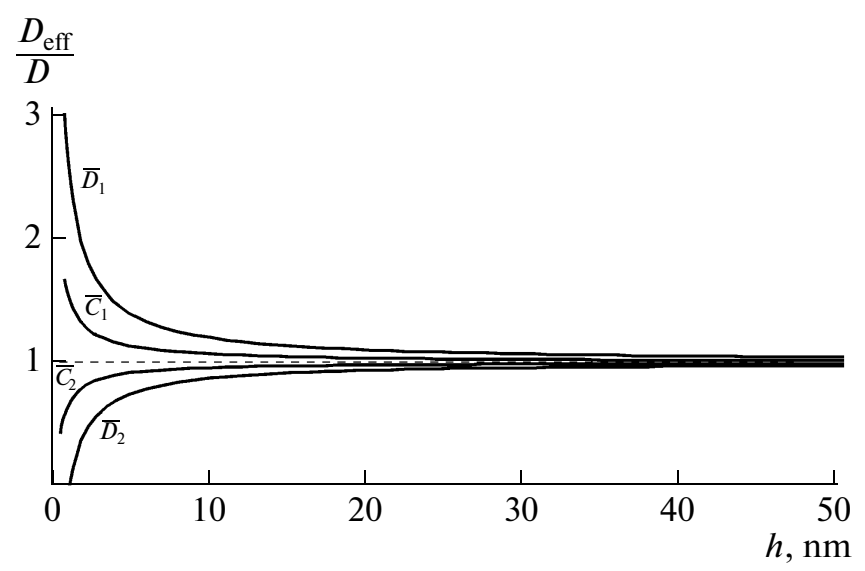

Fig. 3. Dependences of stiffness parameters on thickness.

$$
D \equiv D_{11}+D_{22}=\frac{E h^{3}}{12\left(1-v^{2}\right)},
$$

where $C$ and $D$ are the tangential and bending stiffness parameters, $E$ and $v$ are the Young's modulus and Poisson ratio of the shell material, respectively; and $k$ is the analogue of the transverse-shear factor [15]. The effective tangential and bending stiffness parameters are equal to

$$
\begin{gathered}
C_{\text {eff }} \equiv C_{1}+C_{2}=C+4 \mu_{S}+2 \lambda_{S}, \\
D_{\text {eff }} \equiv D_{1}+D_{2}=D+h^{2} \mu^{S}+\frac{h^{2} \lambda^{S}}{2} .
\end{gathered}
$$

Constitutive equations (14) and (15) make it possible to write the equilibrium equations for the shell and plate with taking into account surface stresses (9) in terms of displacements $\mathbf{w}$ and rotations $\vartheta$. In particular, the equation for the deflection $w=\mathbf{w} \cdot \mathbf{i}_{3}$ in the case of the plate $\left(\mathbf{n}=\mathbf{i}_{3}\right)$ can be reduced to the form

$$
\begin{gathered}
D_{\text {eff }} \Delta \Delta w=\nabla_{S} \cdot \mathbf{m}-\frac{D_{\text {eff }}}{\Gamma} \Delta q_{n}+q_{n}, \\
q_{n}=\mathbf{q} \cdot \mathbf{i}_{3}, \quad \Delta=\nabla_{S} \cdot \nabla_{S} .
\end{gathered}
$$

For the quantitative estimate of the results of the surface-stress effect, we use the data for aluminum [1]: $\mu=34.7 \mathrm{GPa}, \nu=0.3, \lambda^{S}=-3.48912 \mathrm{~N} / \mathrm{m}$, and $\mu^{S}=$ $6.2178 \mathrm{~N} / \mathrm{m}$. We consider the dependences of $D_{\text {eff }}, C_{1}$, $C_{2}, D_{1}$, and $D_{2}$ on the thickness $h$. The plot for the bending stiffness $D_{\text {eff }}$ is shown in Fig. 2, and the plots for the dimensionless values $\bar{C}_{1}=\frac{C_{1}}{C(1-v)}, \bar{C}_{2}=\frac{C_{2}}{C v}$, $\bar{D}_{1}=\frac{D_{1}}{D(1-v)}$, and $\bar{D}_{2}=\frac{D_{2}}{D v}$ are shown in Fig. 3 .

As it follows from Figs. 2 and 3, the surface-stress effect is almost negligible for $h>50 \mathrm{~nm}$. They render the greatest effect for $h<20 \mathrm{~nm}$. In addition, it can be seen that the surface stresses differently affect the stiffness parameters - some of them increase $\left(C_{1}, D_{1}\right)$, 
while others $\left(C_{2}, D_{2}\right)$ decrease, the thicknesses being zero for certain values. Condition (5) guarantees a positive sign for $C_{\text {eff }}$ and $D_{\text {eff }}$ for arbitrary values of $h$.

Thus, we obtained the two-dimensional equilibrium equations for plates and shells with taking into account the transverse shear and the presence of surface stresses. We presented the relations for the stress resultants and couples tensors and found the expressions for effective stiffness parameters of shells. In particular, it was shown that the plate stiffness substantially changes with taking into account the surface stresses, which agrees with the results of the theoretical analysis and the experimental data known in the literature (see, for example, [1]). In particular, it is shown that the shell bending stiffness substantially grows for the nanometer thicknesses.

\section{ACKNOWLEDGMENTS}

This work was supported by the Russian Foundation for Basic Research, project no. 09-01-00459, and DFG (AL 341/31-1).

\section{REFERENCES}

1. H. L. Duan, J. Wang, and B. L. Karihaloo, Adv. Appl. Mech. 42, 1 (2008).

2. E. Orowan, Proc. Roy. Soc. A (London) 316 (1527), 473 (1970).
3. M. E. Gurtin and A. I. Murdoch, Arch. Rat. Mech. Anal. 57 (4), 291 (1975).

4. Ya. S. Podstrigach and Yu. Z. Povstenko, Introduction in Mechanics of Surface Phenomena in Deformable Solids (Naukova Dumka, Kiev, 1985) [in Russian].

5. D. J. Steigmann and R. W. Ogden, Proc. Roy. Soc. A (London) 455 (1982), 437 (1999).

6. K. Dahmen, S. Lehwald, and H. Ibach, Surf. Sci. 446 (1-2), 161 (2000).

7. R. E. Miller and V. B. Shenoy, Nanotecnology 11 (3), 139 (2000).

8. J. G. Guo and Y. P. Zhao, J. Appl. Physics 98 (7), 074306 (2005).

9. P. Lu, L. H. He, H. P. Lee, and C. Lu, Int. J. Solids Struct. 43 (16), 4631 (2006).

10. D. W. Huang, Int. J. Solids Struct. 45 (2), 568 (2008).

11. C. F. Lu, C. W. Lim, and W. Q. Chen, Int. J. Solids Struct. 46 (5), 1176 (2009).

12. V. A. Eremeyev, H. Altenbach, and N. F. Morozov, Dokl. Phys. 54 (2), 98 (2009) [Dokl. Akad. Nauk 424 (5), 618 (2009)].

13. V. A. Eremeyev and L. M. Zubov, Mechanics of Elastic Shells (Nauka, Moscow, 2008) [in Russian].

14. V. V. Novozhilov, K. F. Chernykh, and E. I. Mikhaĭlovskiŭ, Linear Theory of Shells (Politekhnika, Leningrad, 1991) [in Russian].

15. P. A. Zhilin, Applied Mechanics: Fundamentals of Theory of Shells (Izd. Politekhn. Univ., St. Petersburg, 2006) [in Russian].

Translated by V. Bukhanov 